\title{
Pulsed power accelerator for material physics experiments
}

\author{
D. B. Reisman, ${ }^{1}$ B. S. Stoltzfus, ${ }^{1}$ W. A. Stygar, ${ }^{1}$ K. N. Austin, ${ }^{1}$ E. M. Waisman, ${ }^{1}$ R. J. Hickman, ${ }^{1}$ \\ J.-P. Davis, ${ }^{1}$ T. A. Haill, ${ }^{1}$ M. D. Knudson, ${ }^{1}$ C. T. Seagle, ${ }^{1}$ J. L. Brown, ${ }^{1}$ D. A. Goerz, ${ }^{2}$ \\ R. B. Spielman, ${ }^{3}$ J. A. Goldlust, ${ }^{4}$ and W. R. Cravey ${ }^{5}$ \\ ${ }^{1}$ Sandia National Laboratories, Albuquerque, New Mexico 87185, USA \\ ${ }^{2}$ Goerz Engineering Solutions, LLC, Green Bay, Wisconsin 54313, USA \\ ${ }^{3}$ Idaho State University, Pocatello, Idaho 83201, USA \\ ${ }^{4}$ Dielectric Sciences, Inc., Chelmsford, Massachusetts 01824-3526, USA \\ ${ }^{5}$ Alpha-Omega Power Technologies, Albuquerque, New Mexico 87185, USA
}

(Received 12 June 2015; published 9 September 2015)

\begin{abstract}
We have developed the design of Thor: a pulsed power accelerator that delivers a precisely shaped current pulse with a peak value as high as 7 MA to a strip-line load. The peak magnetic pressure achieved within a 1-cm-wide load is as high as $100 \mathrm{GPa}$. Thor is powered by as many as 288 decoupled and transittime isolated bricks. Each brick consists of a single switch and two capacitors connected electrically in series. The bricks can be individually triggered to achieve a high degree of current pulse tailoring. Because the accelerator is impedance matched throughout, capacitor energy is delivered to the strip-line load with an efficiency as high as $50 \%$. We used an iterative finite element method (FEM), circuit, and magnetohydrodynamic simulations to develop an optimized accelerator design. When powered by 96 bricks, Thor delivers as much as 4.1 MA to a load, and achieves peak magnetic pressures as high as $65 \mathrm{GPa}$. When powered by 288 bricks, Thor delivers as much as $6.9 \mathrm{MA}$ to a load, and achieves magnetic pressures as high as $170 \mathrm{GPa}$. We have developed an algebraic calculational procedure that uses the single brick basis function to determine the brick-triggering sequence necessary to generate a highly tailored current pulse time history for shockless loading of samples. Thor will drive a wide variety of magnetically driven shockless ramp compression, shockless flyer plate, shock-ramp, equation of state, material strength, phase transition, and other advanced material physics experiments.
\end{abstract}

DOI: 10.1103/PhysRevSTAB.18.090401

PACS numbers: 84.70.+p, 84.60.Ve

\section{INTRODUCTION}

To generate a magnetic pressure of $100 \mathrm{GPa}$ on the surface of a conductor requires a 500-T magnetic field. Such a field is generated by a linear current density (on the conductor's surface) of $400 \mathrm{MA} / \mathrm{m}$. The first use of such current densities to generate magnetic pressures to drive material-physics experiments is described in the literature $[1,2]$. This magnetically driven technique was developed on the $Z$ accelerator at Sandia National Laboratories.

The technique has been used over the past 15 years for a variety of material studies. Currently the refurbished $Z$ accelerator (also referred to as ZR) produces tailored current pulses to drive samples quasi-isentropically to pressures as high as $500 \mathrm{GPa}$ over timescales as long as $1 \mu \mathrm{s}[3,4]$.

The success of ZR and the shockless compression technique has motivated experimentalists to develop smaller machines that have greater shot throughput and are more economical to operate. Several machines have

Published by the American Physical Society under the terms of the Creative Commons Attribution 3.0 License. Further distribution of this work must maintain attribution to the author(s) and the published article's title, journal citation, and DOI. been built with this aim. Typically, they are low inductance designs that transfer current to strip-line loads to maximize magnetic pressure. As of this writing, machines such as GEPI and Veloce produce currents of 3-4 MA over a near linear rise time of approximately $500 \mathrm{~ns}[5,6]$. In the case of Veloce, this provides a capability of performing shockless compression up to a peak pressure of $\sim 20 \mathrm{Gpa}$.

In this article we describe the design of Thor, a nextgeneration pulsed power accelerator that achieves pressures as high as $100 \mathrm{GPa}$. At such pressures, the Hugoniot and isentrope for most materials diverge; hence 100-GPa class experiments are extremely interesting and useful for equation of state studies. Thor also provides a precise pulse tailoring capability which enables a wide variety of shockless material physics experiments.

The design of the Thor accelerator is outlined in Sec. II. Sections III and IV present simulations of several Thor experiments. A new algebraic technique that calculates the switch triggering sequence necessary to achieve a desired load current time history is described in Sec. V. A discussion of the results is presented in Sec. VI.

\section{THOR CONFIGURATION}

To realize the benefits of an inexpensive isentropic compression experiment (ICE) driver with a high shot rate 


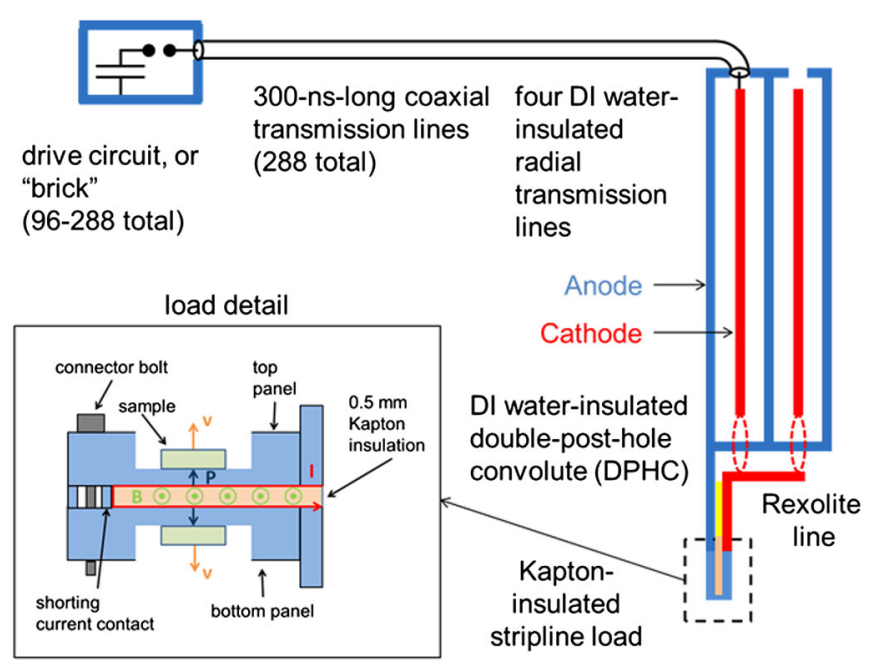

FIG. 1. Conceptual design of Thor. Also shown is the crosssectional ICE load detail depicting the magnetic pressure exerted on the panels and samples.

capability, we have developed the Thor accelerator. It consists of the following components: (1) 96-288 "bricks" that can be individually triggered; (2) 288 coaxial cables that are impedance matched to the bricks; the cable transittime length is chosen to be $300 \mathrm{~ns}$ in order to avoid wave reflections (3); a central, deionized (DI) water dielectric triplate transmission line that is joined into a single line through a double posthole convolute (DPHC); (4) a solid dielectric (Rexolite) transmission line; and (5) a solid dielectric (Kapton) insulated strip-line load. This conceptual design is shown in Fig. 1. We will initially consider the 96-brick version of Thor that is currently under construction.

Each brick consists of two capacitors, a trigger switch, and cable outputs (Fig 2). The two capacitors are charged to opposite polarities with a gas breakdown switch between them. The two Thor brick capacitors each have a capacitance of $80 \mathrm{nF}$ and are nominally charged to a voltage of $+/-100 \mathrm{kV}$. The Thor brick switch is a low inductance field distortion switch produced by High Current Electronics Institute (HCEI) in Tomsk, Russia [7]. The switch is triggered electrically from an electric trigger generator that provides a $100 \mathrm{kV}$ pulse of rise time of 10-20 ns. Each brick has an inductance of approximately $240 \mathrm{nH}$ and a resistance of $0.37 \Omega$. The individual brick energy is $800 \mathrm{~J}$ with a resonant frequency of $2 \mathrm{MHz}$.

Each brick is approximately impedance matched to output cables which act as constant impedance transmission lines to the central power flow section. The optimal impedance is given by the formula [7]

$$
Z=1.1 \sqrt{\frac{L}{C}}+0.8 R
$$

In this case we find that $Z=3.0 \Omega$. Eight brick boxes are arranged into "brick towers" that are sealed and filled with
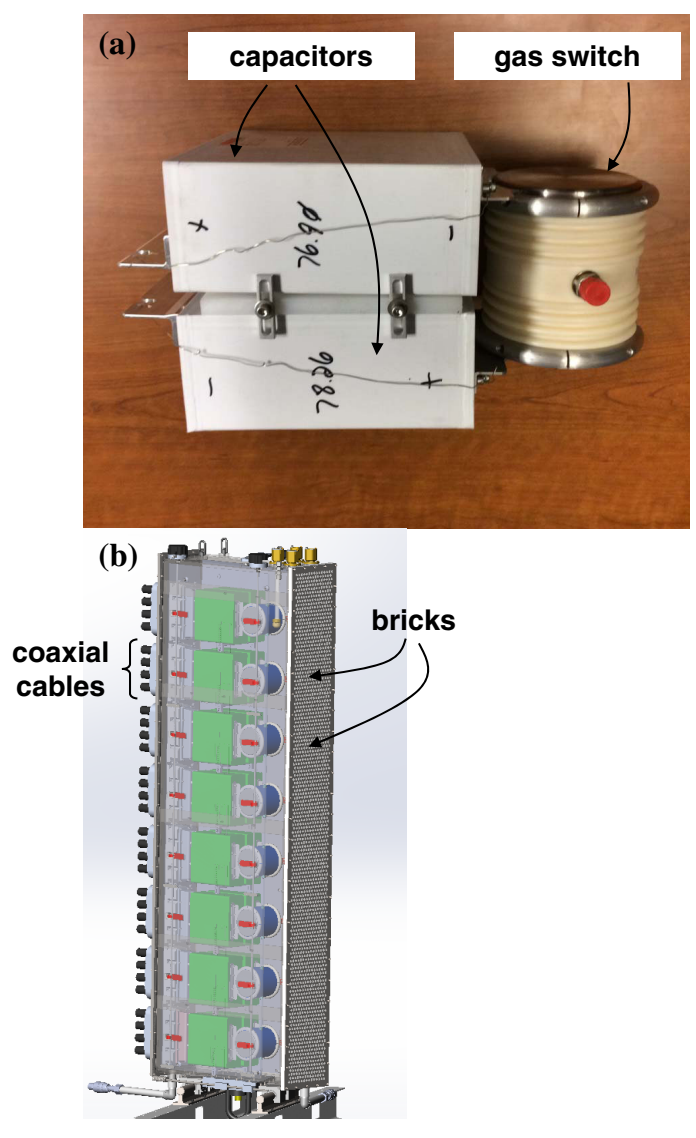

FIG. 2. (a) Individual brick consisting of two capacitors connected to a gas switch. (b) Brick tower consisting of 8 bricks, stacked vertically each with up to 4 cable outputs.

dielectric insulating oil. For the 96-brick version of Thor, we choose to connect three $\sim 10-\Omega$ coaxial cables in parallel to each brick box in order to approximately match the impedance. We note that we have included the option of four cable outputs per brick as future switches and capacitors may be less inductive and consequently produce lower brick impedance. Current for a single brick is shown in Fig. 3. Peak current for $\mathrm{a}+/-100 \mathrm{kV}$ capacitor charge is $36 \mathrm{kA}$ with a rise time of $\sim 60 \mathrm{~ns}$.

Each of these coaxial cables consists of an inner and outer braid of copper conductors, a polyethylene insulator between the conductors, and a thin semiconducting layer on the negative-polarity inner braid. The inner and outer conductors are at radii 1.27 and $1.63 \mathrm{~cm}$, respectively. The overall diameter of the coaxial cable is $3.81 \mathrm{~cm}$, including the outer plastic shield. Each cable is $60 \mathrm{~m}$ long to obtain the $300 \mathrm{~ns}$ transit time required to isolate each brick. The transit time is given by $\tau=l / c$ where $l$ is the cable length and the propagation speed is given by $c=1 / \sqrt{\mu \varepsilon}$. The impedance was initially estimated to be approximately $10 \Omega$. An accurate determination of cable parameters was later made with vector network analyzer (VNA) techniques.

The 288 cables from the 96 bricks are connected to a 2-m diameter central power flow $(\mathrm{CPF})$ structure consisting of 


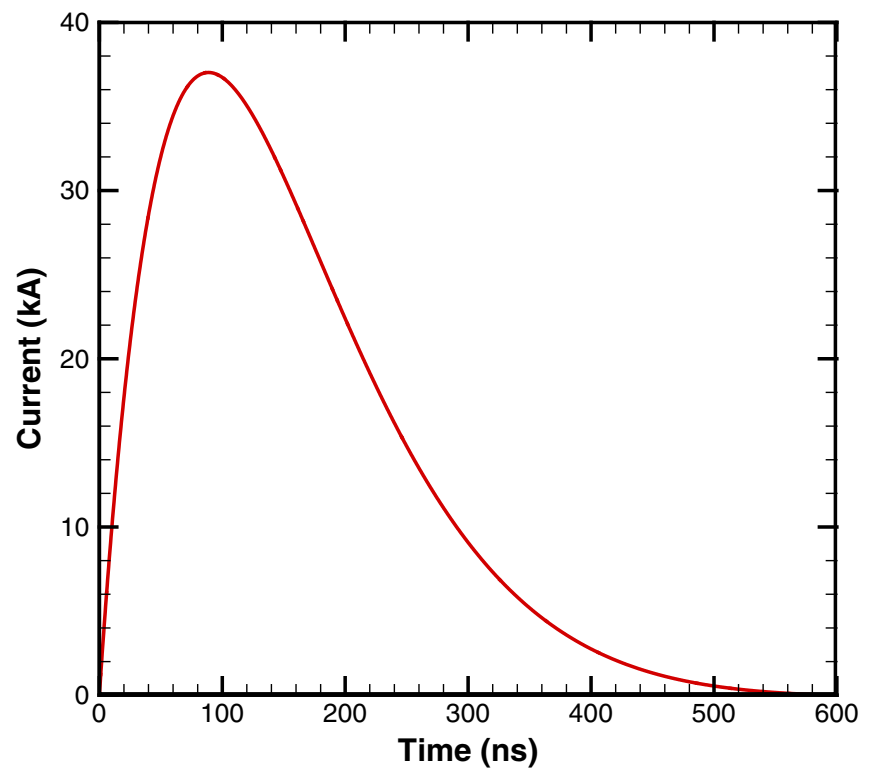

FIG. 3. Current versus time for a single brick into $310 \Omega$ cables. Equivalent total cable impedance is $3.3 \Omega$ and brick impedance is $3.0 \Omega$. Initial capacitor charge is $+/-100 \mathrm{kV}$.

four, DI water-insulated radial transmission lines with an anode-cathode (AK) gap of $1 \mathrm{~cm}$ (Fig. 4). The current from these four parallel lines is connected into a single line using a 40-post DPHC structure. The remaining line is insulated with a layer of Rexolite which varies in AK gap length of $1 \mathrm{~cm}$ at large radius down to a gap of $0.1 \mathrm{~cm}$. This line then transitions to a $0.5-\mathrm{mm}$ thick Kapton-insulated strip line within the central load section.

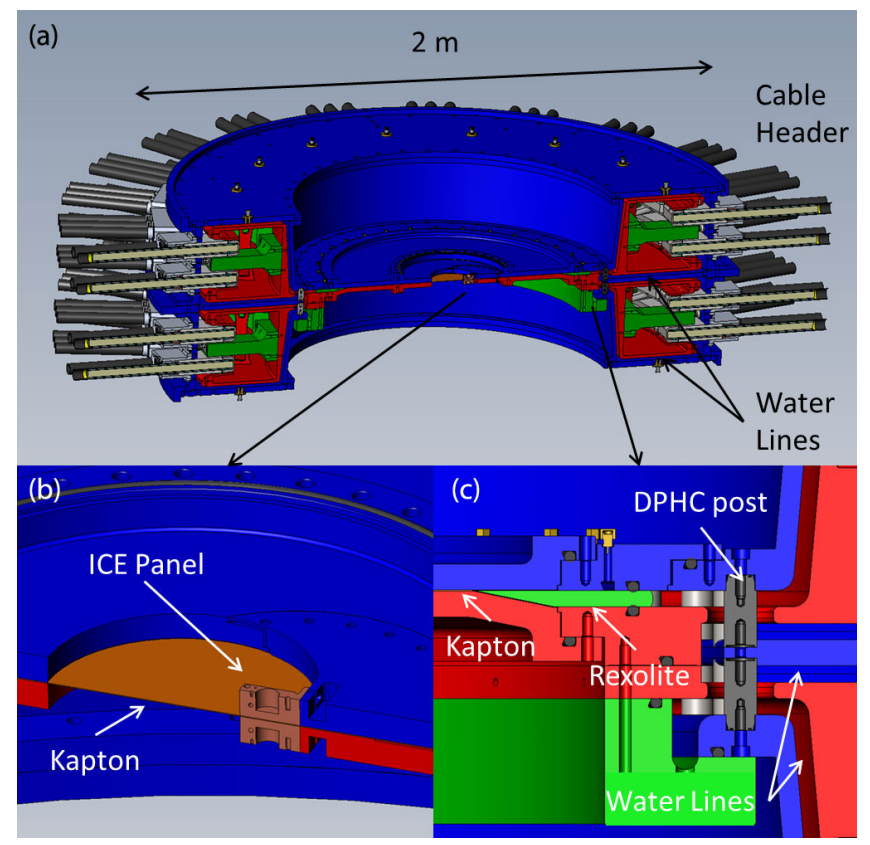

FIG. 4. (a) Cutaway view of the CPF. (b) View of ICE panel in the center load region. (c) Cable header and DI water line section.



FIG. 5. Thor facility showing brick towers and CPF on stand. Cable runs are not shown.

Typical strip-line load panels are 1.0 to $2.0 \mathrm{~cm}$ in width with a length approximately equal to twice the width. This factor of 2 aspect ratio is chosen to ensure uniform pressure drive over the sample region. Panels with width as narrow as $0.5 \mathrm{~cm}$ have been fielded on strip-line generators, but special care must be taken to manage pressure-driven edge waves that can interfere with sample measurement. Load panels are made of either copper or aluminum and are separated by a Kapton-layered film package of total width $0.5 \mathrm{~mm}$.

The full Thor facility is shown in Fig. 5. The diameter of the CPF is motivated by space constraints at future facilities. In fact, the CPF is currently designed to fit within an enclosure at the Dynamic Compression Sector at Argonne National Laboratory. If installed there, Thor may be used to perform time-resolved, in situ x-ray diffraction and imaging measurements of materials under dynamic compression [8].

\section{SIMULATION OF 96-BRICK THOR}

Iterative FEM, circuit, and MHD simulations were used to determine the final Thor design. Initially, estimates of circuit elements were determined using analytic formulas for impedance and inductance. For instance, the impedance $(Z)$ and inductance $(L)$ of the axisymmetrical geometry of the $\mathrm{CPF}$ are given by

$$
\begin{aligned}
& Z=\sqrt{\frac{\mu_{0}}{\varepsilon_{0} \varepsilon_{R}}} \frac{g}{2 \pi r}, \\
& L=2 g \ln \left(\frac{r}{r_{0}}\right),
\end{aligned}
$$

where $r$ is the radius, $r_{0}$ is the inner radius, $g$ is the electrode gap, and $\varepsilon_{R}$ is the dielectric constant. 
Later, these estimates were improved using FEM calculations. Circuit calculations were then performed to determine baseline performance and voltage thresholds for FEM field calculations. Finally, MHD calculations were performed to obtain the dynamic load inductance, a large effect in these systems. With this complete set of circuit parameters and voltage conditions, load performance can be calculated along with the electric field breakdown thresholds of the CPF. We will summarize the final results of this process in this section.

We assume a 96-brick arrangement driving the aforementioned CPF with a strip-line load consisting of $1-\mathrm{cm}$ wide by $2-\mathrm{cm}$ long copper panels. Circuit calculations were performed with the Screamer code $[9,10]$. Each part of the Thor circuit is included in the model as either an inductorresistor-capacitor (LRC) element or a transmission line. Cable attenuation was included as a resistance based on VNA measurements of the coaxial cable. Code outputs are current, energy, power, and voltage for each model element. Brick LRC values are given by manufacturer specifications and laboratory tests for similar configurations.

Peak current is calculated to be 4.1 MA with $35 \mathrm{~kJ}$ of energy delivered to the static strip-line load. In this calculation, 4 groups of 50 capacitors are triggered at staggered intervals to achieve an $200 \mathrm{~ns}$ rise time. All capacitors are initially charged to a voltage of $+/-100 \mathrm{kV}$. The peak voltage delivered to the cables is $120 \mathrm{kV}$. Peak voltages in the DI waterline section and the Rexolite insulator are 100 and $85 \mathrm{kV}$, respectively. The load voltage which is applied across the strip-line Kapton insulated gap is $60 \mathrm{kV}$ (Fig. 6).

The CPF section is modeled using the COMSOL Multiphysics ${ }^{\circledR}$ simulation code [11]. The code uses the FEM to solve for the electric and/or magnetic fields in

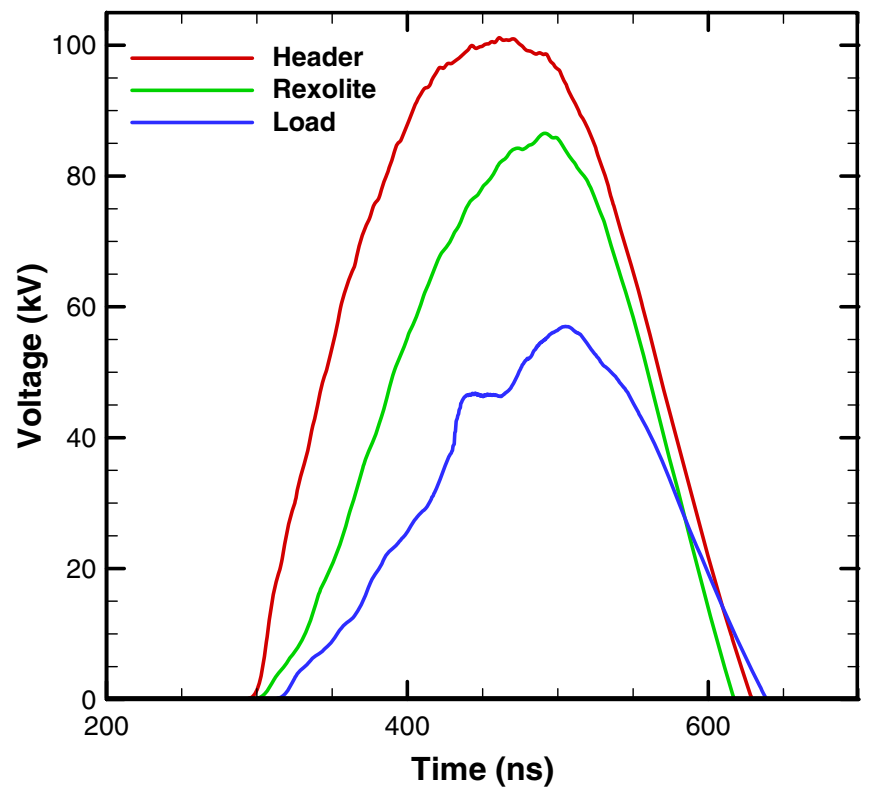

FIG. 6. Voltage waveforms for various regions of the CPF. static, frequency dependent, or transient mode. First, we use the static electric field mode to calculate the electric field distributions driven by the peak voltages calculated with the circuit code. Second, we use the frequency dependent magnetic field solver to calculate the inductance of the convolute structure and the impedance of the cables. Lastly, we calculate the electric field distributions in the cable header region.

Although the CPF section extends out to a diameter of $2 \mathrm{~m}$, we consider only the inner $1.4 \mathrm{~m}$ diameter of the structure (Fig. 7). All the important transitions occur within that radius and the inductance beyond that radius can be easily estimated with Eq. (3) or a separate calculation for the cable header-DI water line section. Using the peak voltages from the previous section, we calculate the electric field distributions. It is critical to the design to maintain an electrical field strength that is below the known electrical field breakdown thresholds. Special attention must also be paid to the triple-point regions where fields can be enhanced due to disparities in dielectric constants across material boundaries.

For our three insulator materials-DI water, Rexolite, and Kapton-we determine the electric field strength thresholds. For a DI water-insulated line this is given empirically by the formula $[12,13]$

$$
E_{P}=108 \tau_{\mathrm{EFF}}^{-0.33}(\mathrm{kV} / \mathrm{cm})
$$

where $E_{P}$ is the peak electrical field and $\tau_{\mathrm{EFF}}$ is the temporal width (in $\mu \mathrm{s}$ ) of the voltage pulse at $63 \%$ of peak. In our case $\tau_{\mathrm{EFF}}=200 \mathrm{~ns}$ results in $E_{P}=184 \mathrm{kV} / \mathrm{cm}$. The Rexolite breakdown threshold has been determined to be $\sim 800 \mathrm{kV} / \mathrm{cm}$ while layered Kapton has a threshold of $\sim 2000 \mathrm{kV} / \mathrm{cm}$.

Assuming an $85 \mathrm{kV}$ voltage across the AK gap as the boundary condition, we calculate the electric fields in the CPF (Fig. 8). Slice contour plots of electric field magnitude

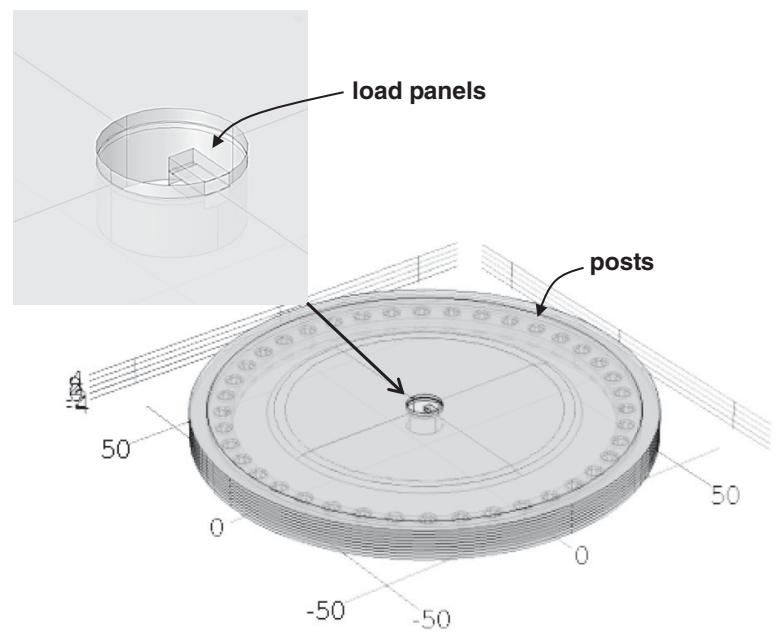

FIG. 7. 3D COMSOL computational setup for calculation of CPF magnetic and electric fields. 


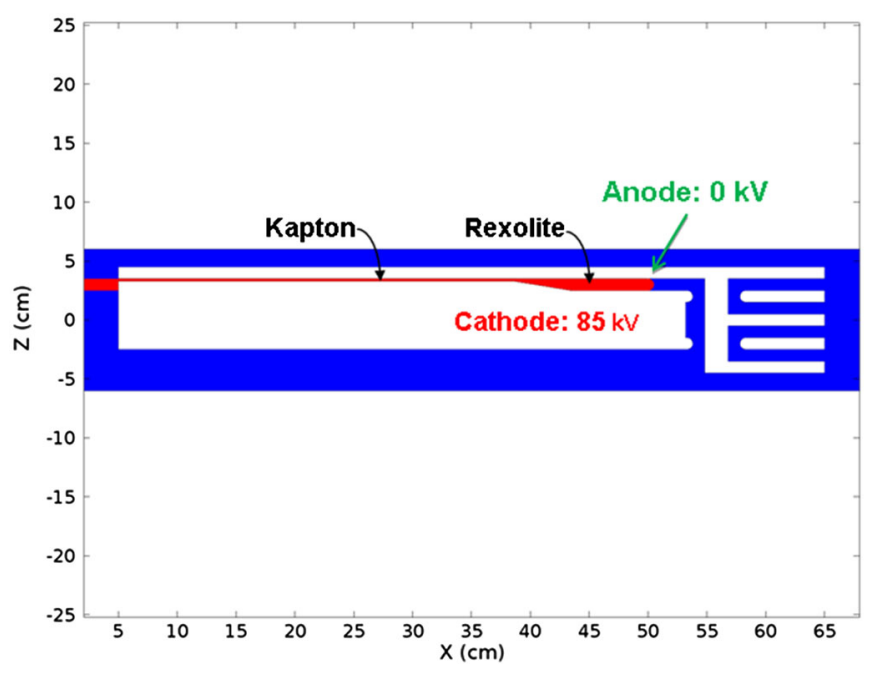

FIG. 8. Cutaway view of DPHC structure in calculation of electric fields. Shown are the boundary voltage values used for the electric field calculations.

are shown in Fig. 9. Note that we taper the Rexolite insulator from the DI water-line gap thickness $(10 \mathrm{~mm})$ to the final value $(1 \mathrm{~mm})$ in order to maintain electric fields at the Rexolite-DI water interface that are below the DI water dielectric thresholds. In the DI water region, the maximum electric field is $\sim 100 \mathrm{kV} / \mathrm{cm}$, which is well below our threshold of $184 \mathrm{kV} / \mathrm{cm}$. In the insulator region, the field increases to $\sim 500 \mathrm{kV} / \mathrm{cm}$ in the Rexolite before transitioning to the Kapton which is at $\sim 1700 \mathrm{kV} / \mathrm{cm}$. Both of these values are below the previously mentioned Rexolite and Kapton breakdown thresholds.

Having established sufficient gaps for operating the inner power flow structure, we calculate the inductance of the system. We use a frequency-dependent magnetic field calculation in order to capture skin depth effects on the inductance. Assuming an input current of $8 \mathrm{MA}$ at a frequency of $2 \mathrm{MHz}$, we calculate the magnetic field distribution in the central power flow region. Slice views of the magnetic field magnitude show concentrations of fields in the posts and in the strip-line load region where the entire field is concentrated (Fig 10). A peak magnetic field of $\sim 800 \mathrm{~T}$ is obtained in the 1-cm-wide strip line which corresponds to a magnetic pressure of $250 \mathrm{GPa}$. It should be noted that this calculation is static. In the actual experiment, the plates will separate due to the Lorentz force, increasing the gap and reducing the fields and pressures due to edge effects. Inductance of the structure is calculated through the energy relation $L=\sqrt{2 E_{M}} / I$ where $E_{M}$ is the magnetic energy. For this particular strip-line load arrangement, the static inductance is found to be $2.5 \mathrm{nH}$. Including the remainder of the $\mathrm{CPF}$ structure we obtain a total inductance of $3.0 \mathrm{nH}$.

Coaxial cable impedance was modeled with the COSMOL simulation code. To determine impedance, the coax is
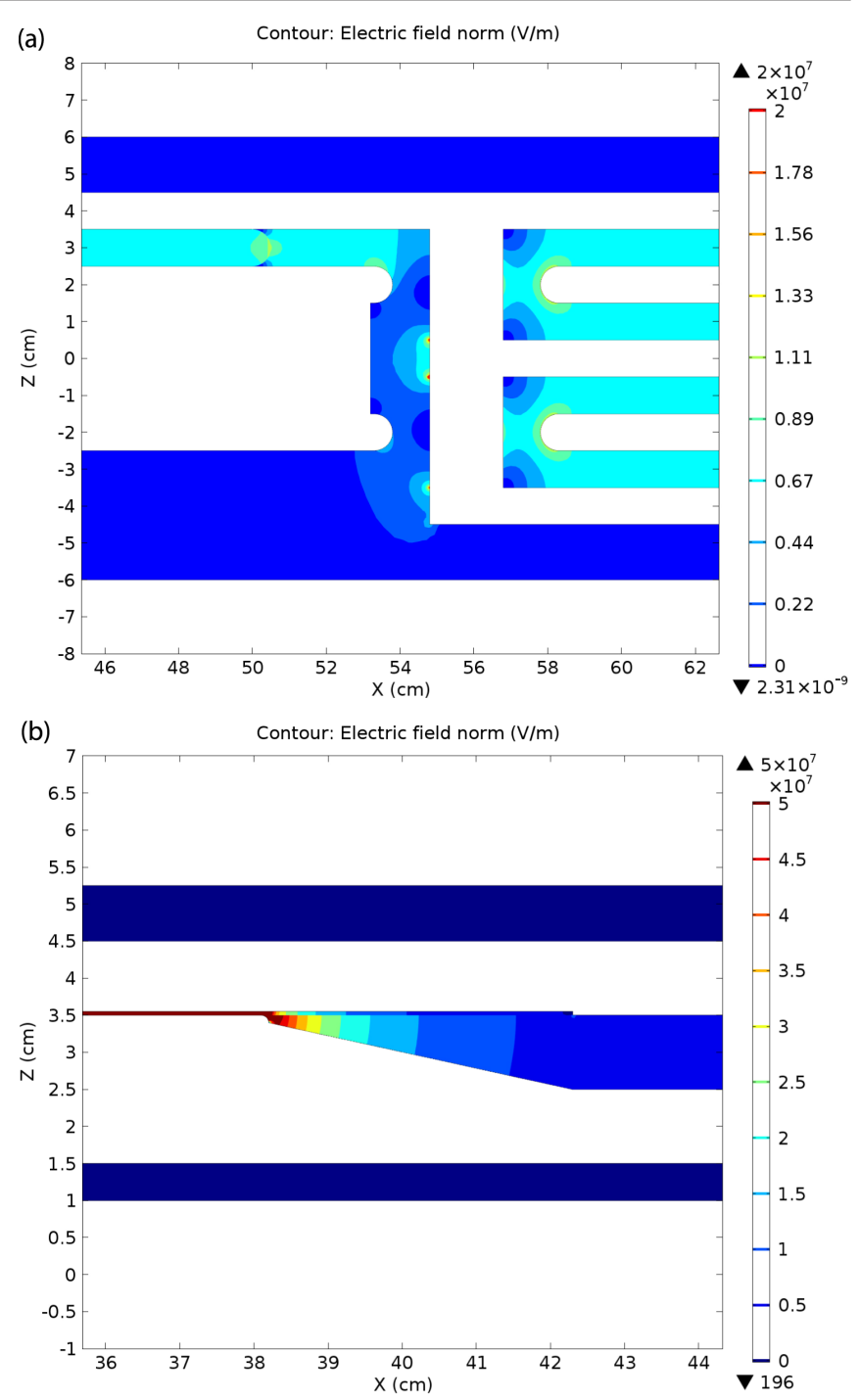

FIG. 9. (a) Close-up view of electric fields in critical DI waterrexolite transition. (b) Rexolite-Kapton interface.

modeled as a two-dimensional cross section of a TEM waveguide. First, the electric and magnetic field distributions at a frequency of $2 \mathrm{MHz}$ are calculated (Fig. 11). It should be noted that the semiconducting layer pushes the electric field away from the inner conductor, a desired effect to prevent breakdown coronas on the cathode. Second, line integrals of electric and magnetic field are used to calculate voltage and current,

$$
\begin{gathered}
V=V_{i}-V_{o}=-\int_{R_{o}}^{R_{i}} E \cdot d r, \\
I=\frac{1}{\mu_{0}} \oint_{C} B \cdot d l,
\end{gathered}
$$

where the subscripts $i$ and $o$ denote inner and outer conductor quantities. Finally, the characteristic impedance 

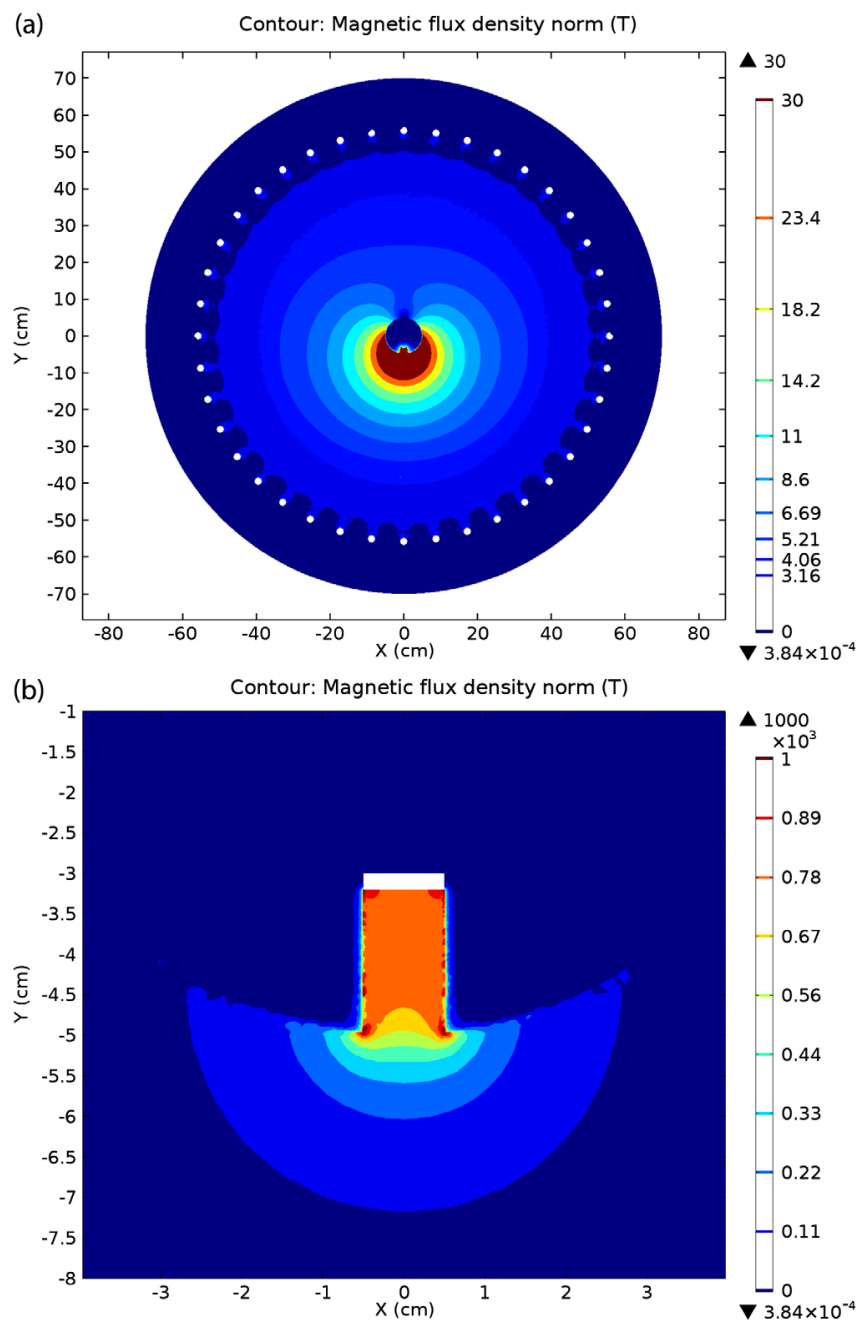

FIG. 10. Contour plots of magnetic field in CPF. (a) Cutaway view of field in AK gap from outer convolute to load. (b) Concentration of fields in strip line.

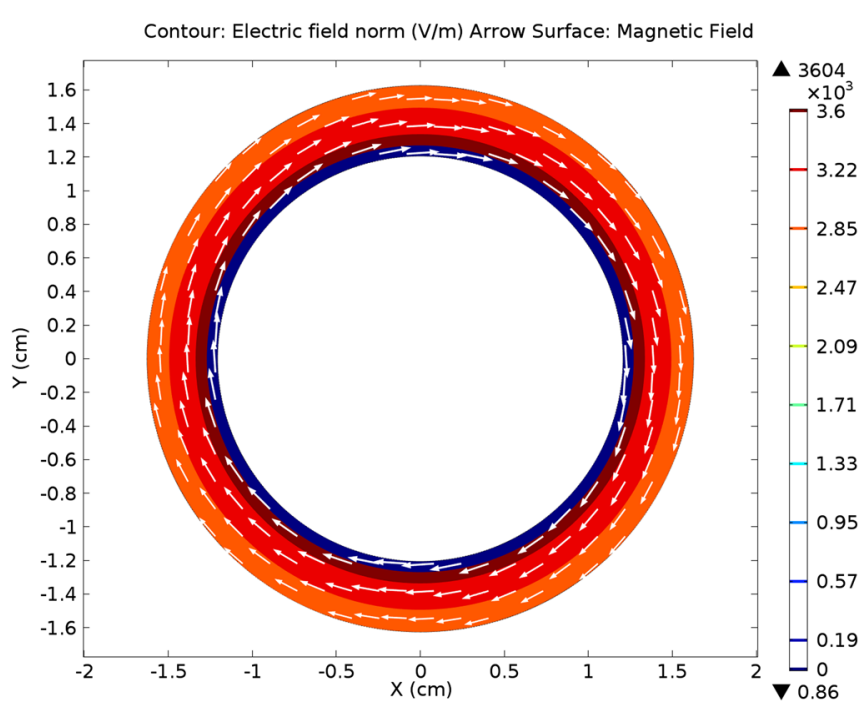

FIG. 11. Radio-frequency calculation of the electromagnetic fields in a cross section of coaxial cable. White lines are magnetic field vectors. Contours are electric fields.

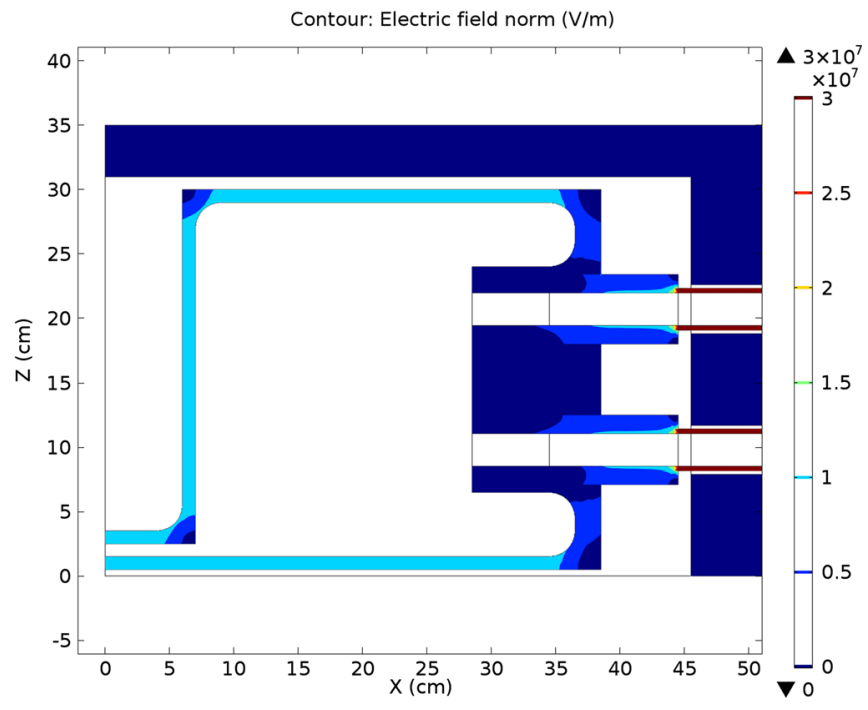

FIG. 12. Contour plot of electric field in cable header-DI water line region.

is calculated through the relation $Z=V / I$. The impedance of the cable is found to be $10.5 \Omega$ at a frequency of $2 \mathrm{MHz}$; VNA measurements determined the inductance to be $10.8 \Omega$.

The cable connection region was modeled to determine if any electrical breakdown thresholds were exceeded. Because of the periodic nature of the cable connection region to the central power flow, only two cables were modeled on half of the $\mathrm{CPF}$ cable header region. Mirror boundary conditions were imposed to accurately represent a section of the geometry. As in the modeling described earlier, a voltage of $100 \mathrm{kV}$ was applied across the AK gap as a boundary condition. Contours of electric field show

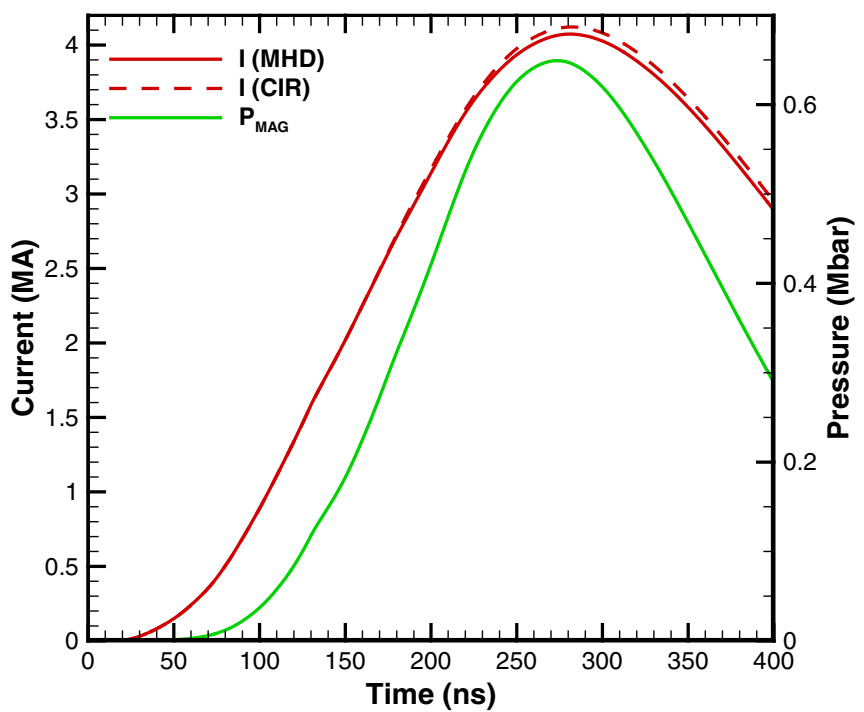

FIG. 13. Current and magnetic pressure for 96-brick Thor. Current is computed in both Trac-II MHD code and in the Screamer circuit code. 
concentrations in the region of cable "breakout" where the center conductor of the coaxial cable enters the DI water dielectric (Fig. 12). The field in the break-out region is managed by introducing a Delrin plug that reduces the electrical field at the poly-DI water interface. By doing this the peak electric field can be reduced to be below the breakdown threshold of DI water.

MHD calculations were performed to help determine the full system electrical performance, and peak pressures delivered to a strip line load. Because the inductance of the load at the time of peak pressure is significant relative to the total inductance, an accurate estimate of delivered current must include the dynamic inductance of the strip line. Trac-II, a resistive 2D MHD code, was used to model a cross section of the strip line [1]. We assume that 3D effects are small and can be neglected. The Screamer circuit code was used to obtain the load drive voltage which was applied as an input to the Trac-II simulations. Self-consistency

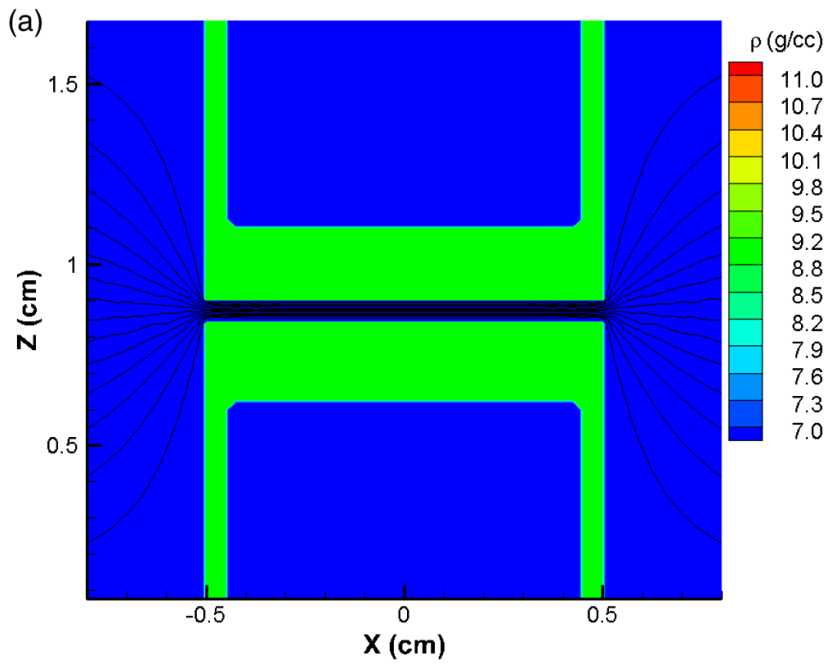

(b)

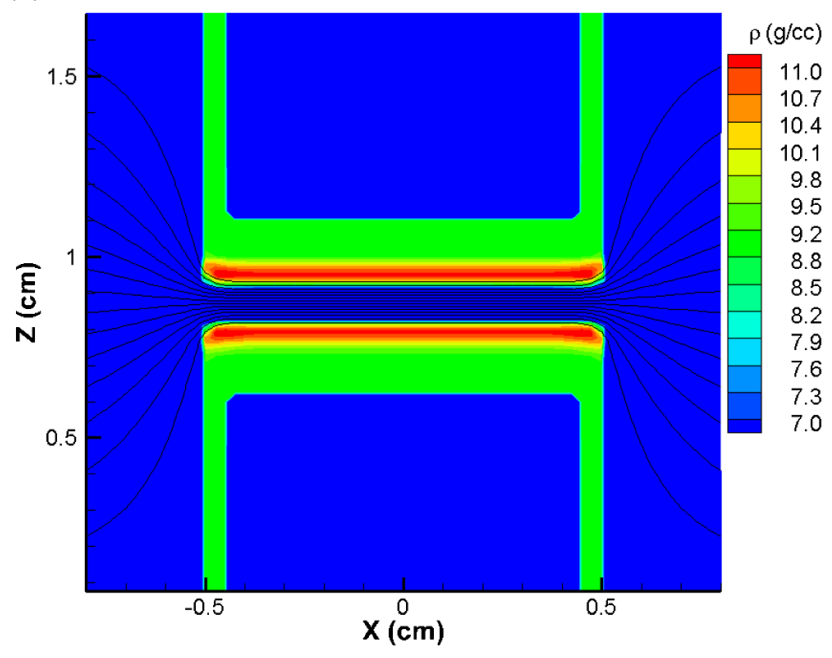

FIG. 14. Contour plots of density in ICE panels at (a) 0 and (b) $300 \mathrm{~ns}$ (peak pressure). Lines are magnetic flux. was obtained by using the time-dependent inductance from the MHD calculation in the circuit code to obtain an improved drive voltage. This process was repeated until agreement in voltage and current was obtained between Screamer and Trac-II.

Pulse tailoring was obtained by triggering four groups of capacitors at staggered $50 \mathrm{~ns}$ intervals. For this particular case, a nearly linear $200 \mathrm{~ns}$ pulse with peak current of 4.1 MA produced a peak magnetic pressure of $65 \mathrm{GPa}$ (Fig. 13). During the pulse the strip-line load inductance increases from 1.2 to $2.3 \mathrm{nH}$ as the panels are compressed and separate (Fig. 14).

\section{EXTENSION TO 144- AND 288-BRICK THOR}

Several options exist for extending the present design to well beyond 96 bricks and increasing the peak current and pressure at the ICE load. One of these is to simply increase the diameter of the CPF to allow more of the $10.8 \Omega$ cables to be connected and therefore more bricks to be added to the system. A more challenging but more attractive option is to lower the impedance of the individual cables and allow more cable connections while maintaining the impedance matching conditions between brick and cable. For instance, a $3.0 \Omega$ cable would allow one cable per brick and result in up to 288 bricks to be attached to the CPF.

In order to reduce the impedance of the cable while maintaining its approximate diameter and voltage hold-off properties, the dielectric constant (or relative permittivity) of the insulating material must be increased. The impedance of a coaxial cable is given by

$$
Z=\frac{1}{2 \pi} \sqrt{\frac{\mu_{0}}{\varepsilon_{0} \varepsilon_{R}}} \ln \left(\frac{R_{o}}{R_{i}}\right) .
$$

Since the impedance is proportional to $\varepsilon_{R}{ }^{-1 / 2}$, Thor's present $10.8 \Omega$ cable can be decreased to $3.4 \Omega$ by increasing the dielectric constant from 2.3 (polyethylene) to 21. This also has the advantage of reducing the overall length of each cable while maintaining the 300 ns transit time for isolation purposes. Since the velocity of propagation also varies as $\varepsilon_{R}^{-1 / 2}$, a dielectric constant of 21 would reduce the cable length from 60 to $20 \mathrm{~m}$. This would also have the added benefit of reducing signal attenuation in the cable which is dependent on conductor losses and proportional to length.

We are developing a high dielectric constant polyethylene that would serve as a replacement to the standard polyethylene used in our previously described cable. This is accomplished by impregnating the polyethylene with ceramic nanoparticles. Preliminary efforts have resulted in samples with dielectric constant of 21 , but with reduced dielectric strength. The electric field in a coaxial cable is given by 


$$
E=\frac{1}{\ln \left(\frac{R_{o}}{R_{i}}\right)} \frac{V}{r} .
$$

Assuming a peak voltage of $120 \mathrm{kV}$, the maximum electric field in our present cable is $380 \mathrm{kV} / \mathrm{m}$ on the inner conductor $\left(r=R_{i}\right)$. Testing of the loaded-poly samples revealed a dielectric strength of only $\sim 200 \mathrm{kV} / \mathrm{cm}$. An effort is currently underway to raise this strength to $\sim 400 \mathrm{kV} / \mathrm{cm}$ in samples with a dielectric constant ranging from 9 to 21 . We speculate that reducing the dielectric constant to 9 might result in better dielectric strength, and would result in a 144-brick system with two, $5.4 \Omega$ cables per brick.

Another way to obtain a low-inductance cable based on increasing the dielectric constant is to use deionized DI water as the insulating medium. To evaluate this concept we are testing a $50 \Omega$ air-dielectric cable filled with DI water. The cable is 1-5/8" HJ7-0A Andrew Heliax ${ }^{\circledR}$ manufactured by CommScope, Inc. The cable is used in the telecommunications industry as a low-loss radio-frequency transmission line. It consists of an inner and outer corrugated copper conductor separated by a helical polyethylene spacer. COMSOL calculations indicate that a DI water-filled Heliax would have an impedance of $6.4 \Omega$, ideal for a twocable per brick arrangement. Based on our velocity of propagation arguments, the DI water cable would only need to be $10 \mathrm{~m}$ long to give us the required $300 \mathrm{~ns}$ transit time. If testing of this cable proves to be successful, it could be used to extend Thor to 144 bricks. An increase of the inner conductor radius by $0.5 \mathrm{~cm}$ would decrease the cable inductance to $3.45 \Omega$, making it suitable for a one-cableper-brick, 288-brick Thor system.

The various cable options are summarized in Table 1. We believe that a 144-brick Thor is achievable in the near future either based on loaded poly or DI water-Heliax cable. A 288-brick Thor is possible but might require a longer coaxial cable development path. We consider the performance of both the 144-brick and 288-brick Thor systems using the same modeling approach and load configuration described in the previous section. For the $1-\mathrm{cm}$ wide by $2-\mathrm{cm}$ long panel arrangement and 144-brick Thor, we obtain a peak current of 5.4 MA with a peak pressure of $110 \mathrm{GPa}$. For 288-brick Thor we obtain a peak

TABLE I. Comparison of cable options to expand the number of bricks while maintaining impedance matching between bricks and cable.

\begin{tabular}{lcccccc}
\hline \hline Type of cable & $\varepsilon_{\mathrm{R}}$ & $\begin{array}{c}\mathrm{Ro} \\
(\mathrm{cm})\end{array}$ & $\begin{array}{c}\mathrm{Ri} \\
(\mathrm{cm})\end{array}$ & $\begin{array}{c}\mathrm{Z} \\
(\Omega)\end{array}$ & $\begin{array}{c}\text { Length } \\
(\mathrm{m})\end{array}$ & $\begin{array}{c}\text { Emax } \\
(\mathrm{kV} / \mathrm{cm})\end{array}$ \\
\hline Standard & 2.3 & 1.63 & 1.27 & 10.8 & 60 & 380 \\
Nanopoly & 9 & 1.63 & 1.27 & 5.42 & 30 & 380 \\
Nanopoly & 21 & 1.63 & 1.27 & 3.55 & 20 & 380 \\
DI water & 80 & 2.32 & 0.90 & 6.40 & 10 & 141 \\
DI water-Mod & 80 & 2.32 & 1.40 & 3.45 & 10 & 170 \\
\hline \hline
\end{tabular}

TABLE II. Comparison of load performance for 10-mm-wide panels with 96-, 144-, and 288-brick Thor. Cables are approximately impedance matched to the bricks as described in Sec. IV.

\begin{tabular}{lcccccc}
\hline \hline $\begin{array}{l}\text { Brick } \\
\text { number }\end{array}$ & $\begin{array}{c}\text { Cables } \\
\text { per brick }\end{array}$ & $\begin{array}{c}\text { Total E } \\
(\mathrm{kJ})\end{array}$ & $\begin{array}{c}\text { Peak I } \\
(\mathrm{MA})\end{array}$ & $\begin{array}{c}\text { Peak P } \\
(\mathrm{GPa})\end{array}$ & $\begin{array}{c}\text { Load E } \\
(\mathrm{kJ})\end{array}$ & $\begin{array}{c}\text { Efficiency } \\
(\%)\end{array}$ \\
\hline 96 & 3 & 76.8 & 4.1 & 65 & 27.0 & 35 \\
144 & 2 & 115 & 5.4 & 110 & 56.1 & 49 \\
288 & 1 & 230 & 6.9 & 170 & 111 & 48 \\
\hline \hline
\end{tabular}

current of 6.9 MA with a peak pressure of $170 \mathrm{GPa}$. The results of all three Thor configurations $(96,144$, and 288 bricks) are summarized in Table 2. Note that an increase in efficiency is realized in the 144-brick and 288-brick systems as cable length, and therefore signal attenuation, is reduced.

\section{CIRCUITLESS PULSE TAILORING OF THOR}

As mentioned earlier, pulse tailoring is desired to delay the onset of shocks in samples. Pulse shaping delays the intersection of loading characteristics [5]. Ideally, the pulse shape can be tailored so that all characteristics intersect at a single "critical" location $X c$. Given the equation of state of a material, ideal pressure waveforms can be calculated. On machines such as ZR, pulse tailoring is accomplished by individually triggering gas switches into 36 transmission lines. MHD and transmission line codes are used to determine the sequence to produce the most optimal pulse for a specific experiment. Usually this involves hand-tuning trigger times in a circuit code until the desired waveform is achieved. Critical to the process is an accurate circuit model which, in the case of ZR, contains over 50,000 circuit elements.

For Thor, we can take advantage of the transit time isolation of our bricks and determine our pulse without the use of a circuit or transmission line code. Essentially this involves using the brick current waveforms as basis functions to construct the desired configuration. The forwardgoing power in any of the constant impedance identical coaxial lines is independent of the load, and of the other lines, for the time interval between the earliest triggered brick pulse arrival at the connecting point, and the round trip electromagnetic transit time from that connecting point to that brick and back. Furthermore, given that the load is at a small radius, only the voltage average and the total current of all bricks need to be considered [14]. These observations motivated the development of a procedure, which does not need circuit calculations, and uses the single-brick basis function to determine the brick-triggering sequence necessary to generate a highly tailored current pulse for shockless loading of samples.

Using TEM waveguide theory, we may express the equivalent total voltage and current into forward- and backward-propagating waves 


$$
\begin{aligned}
& V=V_{+}+V_{-}, \\
& I=\frac{V_{+}}{Z}-\frac{V_{-}}{Z} .
\end{aligned}
$$

This can be rewritten in terms of the forward-propagating quantities,

$$
\begin{aligned}
& V_{+}=\frac{1}{2}(V+Z I), \\
& I_{+}=\frac{1}{2}\left(\frac{V}{Z}+I\right) .
\end{aligned}
$$

Since the bricks are transit-time decoupled and the transmission lines are of constant impedance, the forward-going current is the sum of all individual brick currents,

$$
I_{+}=\sum_{k=1}^{N} i_{k}\left(t-\tau_{k}\right)
$$

where $\vec{\tau}=\left(\tau_{1}, \ldots, \tau_{n}\right)$ is the set of brick delay times and $i_{k}$ is the forward-propagating brick current from the $k$ th brick. (An example of an $i_{k}$ waveform is given in Fig. 3.). Using the desired ICE load voltage and current $\left(V_{D}, I_{D}\right)$ and the inductance of the CPF between the load and the cable header $\left(L_{C}\right)$, we may determine our desired forward-going current:

$$
I_{0+}=\frac{1}{2}\left[\frac{V_{D}+L_{C} \dot{I}_{D}}{Z}+I_{D}\right]
$$

Note that for convenience we have lumped the DI water transmission line element of the CPF into the impedance $L_{C}$. This is a reasonable approximation since the water line transit time of $\sim 20 \mathrm{~ns}$ is much shorter than the typical current risetime of 200-500 ns.

We then perform an optimization procedure to determine a set of $\tau_{k}$ such that $I_{0+} \approx I_{+}$. This involves finding a local minimum to the $L_{2}$ norm given by

$$
F(\vec{\tau})=\int_{0}^{T} d t\left[I_{+}(t)-I_{0+}(t)\right]^{2}
$$

In a future publication we will describe the specifics of the minimization algorithm.

As an example we consider our previous ICE load configuration: $1-\mathrm{cm}$ wide by $2-\mathrm{cm}$ long copper strip-line panels. First, we determine the ideal pressure waveform $P_{I}(t)$ for a $\sim 100 \mathrm{GPa}$ maximum pressure and $350 \mathrm{~ns}$ rise time that will ensure shockless loading up to the location $X_{C}=0.24 \mathrm{~cm}$ (Fig. 15). Second, we use the MHD code to iteratively determine the current $I_{D}(t)$ and voltage $V_{D}(t)$ such that the magnetic pressure $P_{\mathrm{MAG}}(t)$ approximates the ideal drive pressure $P_{I}(t)$ :

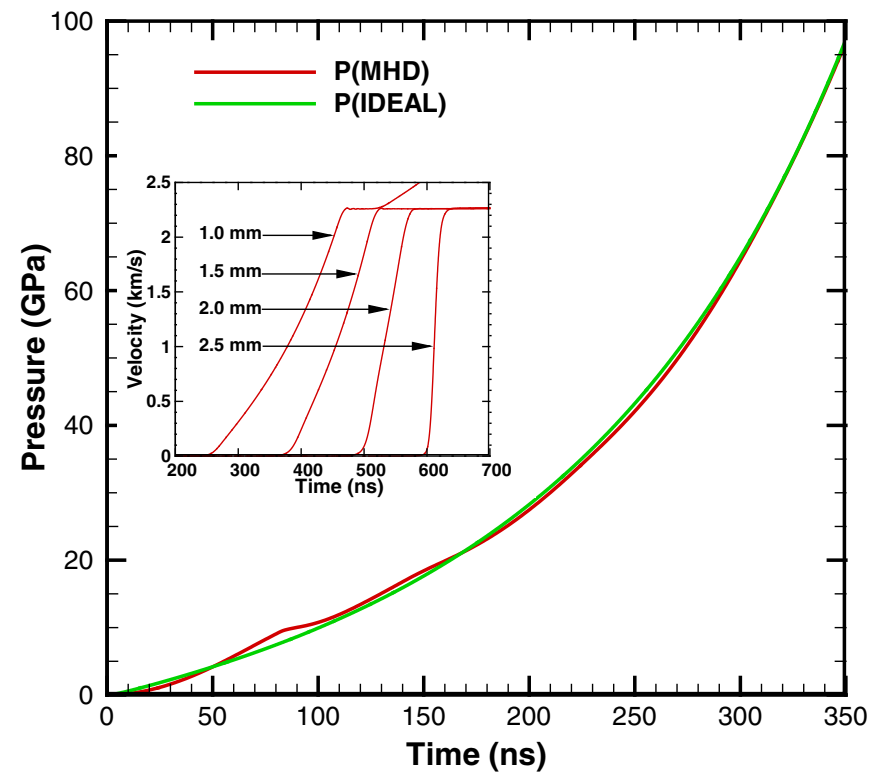

FIG. 15. Ideal and MHD code-calculated pressure drive for shockless compression of copper. Inset shows copper-LiF interface velocities for the tailored pressure pulse. Ramp loading is maintained until sample thickness is $X_{C}=2.4 \mathrm{~mm}$.

$$
P_{I}(t) \approx P_{\mathrm{MAG}}(t)=B^{2}(t) / 2 \mu_{0}
$$

where $B(t)$ is the magnetic field magnitude in the center of the insulating gap between the two drive panels (Fig. 16). Third, using Eq. (14) we calculate the desired forward-going current that appears on the CPF after the TEM wave has propagated through the coaxial transmission lines. In this case, the central power-flow inductance is given by $L_{C}=$ $1.8 \mathrm{nH}$ and the transmission line impedance is given by

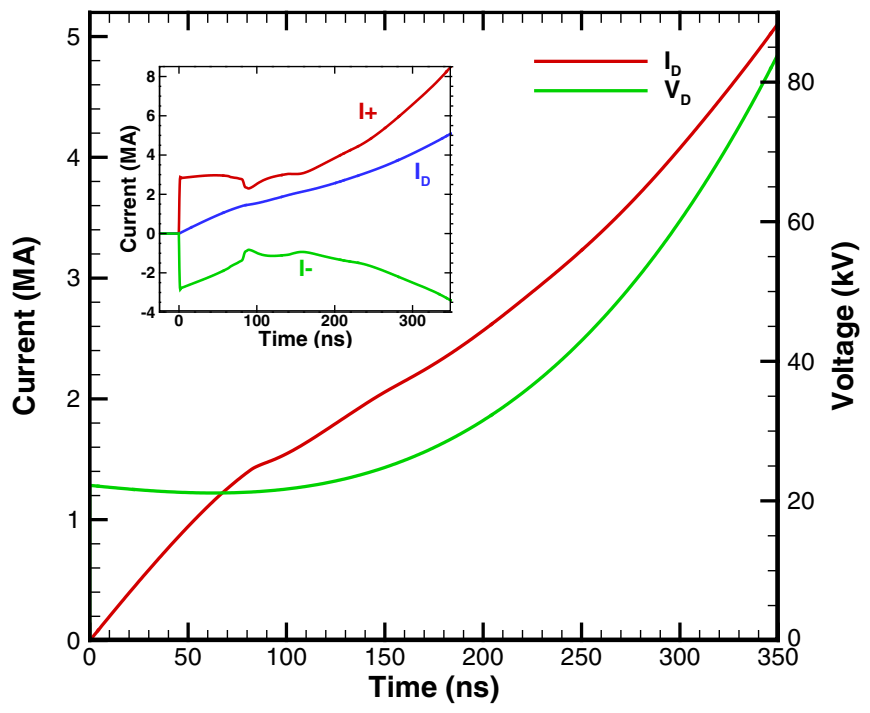

FIG. 16. Desired current and voltage for compression of copper. Inset shows decomposition of current (I) into forwardgoing $\left(\mathrm{I}_{+}\right)$and backward-going $\left(\mathrm{I}_{-}\right)$waves. 


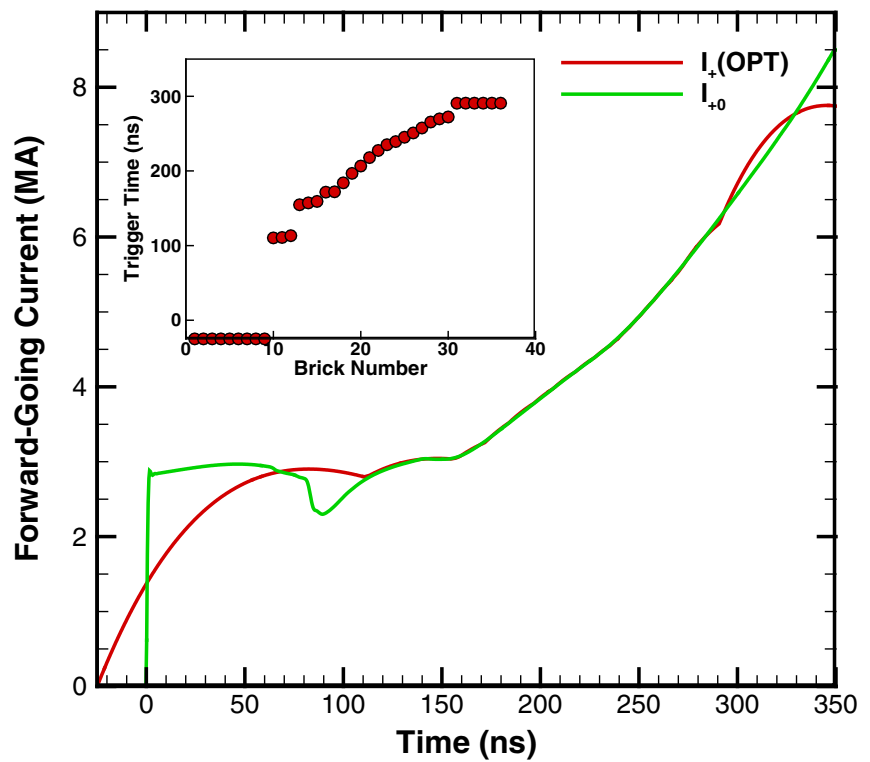

FIG. 17. Optimized fit for forward-going current compared to desired forward-going current. Inset shows trigger times $(\tau)$ for $100 \mathrm{GPa} \mathrm{Cu}$ compression example with 36 independent trigger times and 288 bricks.

$$
Z=Z_{C} / N_{B}
$$

where $Z_{C}$ is the single-brick effective cable impedance and $N_{B}$ is the total number of bricks. Finally, we form $F(\vec{\tau})$ given in Eq. (15), using the appropriate brick basis functions $i_{k}$, and find the local minimum. This in turn gives us the optimal set of delay times $\vec{\tau}$.

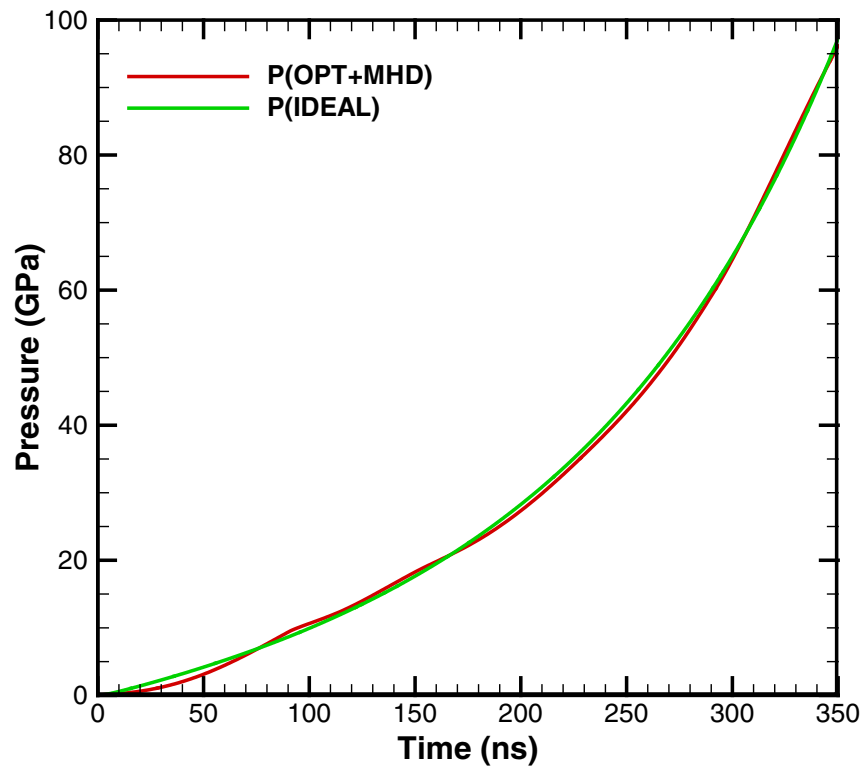

FIG. 18. Pressure profile obtained by using the voltage from the optimization procedure in the MHD code compared to the ideal pressure pulse.
The results of the optimization procedure for a 288-brick system $\left(N_{B}=288\right)$ are shown in Fig. 17. The calculation assumes a perfectly matched single-brick cable impedance of $Z_{C}=3.0 \Omega$ and a maximum capacitor charge of $+/-110 \mathrm{kV}$. A good fit is obtained between the desired forward-going current $I_{0+}$ and the current produced by the optimized linear combination of brick currents $I_{+}(\vec{\tau})$. The results were achieved with 36 independent trigger times. Some difficulty is encountered in matching the rapid earlytime rise of the desired current which is largely a result of the characteristics condition to obtain the ideal isentropicloading condition. However, good agreement is obtained between the pressure waveform produced by the optimization procedure, when computed with the MHD code, and the ideal pressure drive (Fig. 18). Part of the optimization process is determining the number of bricks that are needed to produce the desired current and voltage waveforms. This was ultimately how a 288-brick system was chosen for this one-megabar example. We therefore conclude that one of the strengths of this technique is the ability to evaluate peak-pressure capabilities of proposed Thor configurations as a function of the number and type of bricks.

\section{CONCLUSION}

We have designed Thor, a pulsed power accelerator, for performing magnetically driven, quasi-isentropic compression experiments. The design uses multiple capacitordriven brick switches to deliver current to a power flow structure via impedance-matched, transit-time-isolated coaxial cables. The current is concentrated into a strip-line load where dynamic material experiments can be performed using the large magnetic pressures. Peak pressure depends on the number of bricks and ranges from $65 \mathrm{GPa}$ for a 96-brick Thor to $170 \mathrm{GPa}$ for a 288-brick Thor. Because of the decoupling of the individual bricks, current can be tailored to achieve optimal shockless loading. This is done through a straightforward optimization procedure that does not require a circuit code but uses the brick waveforms as basis functions in building an optimal total current pulse.

\section{ACKNOWLEDGMENTS}

This work was supported in part by a Laboratory Directed Research and Development grant from Sandia National Laboratories. Sandia is a multiprogram laboratory operated by Sandia Corporation, a Lockheed Martin Company, for the United States Department of Energy's National Nuclear Security Administration under Contract No. DE-AC04-94AL85000.

[1] D. B. Reisman, A. Toor, R. C. Cauble, C. A. Hall, J. R. Asay, M. D. Knudson, and M. D. Furnish, J. Appl. Phys. 89, 1625 (2001). 
[2] C. A. Hall, J. R. Asay, M. D. Knudson, W. A. Stygar, R. B. Spielman, T. D. Pointon, D. B. Reisman, A. Toor, and R.C. Cauble, Rev. Sci. Instrum. 72, 3587 (2001).

[3] J.-P. Davis, C. Deeney, M. D. Knudson, R. W. Lemke, T. D. Pointon, and D. E. Bliss, Phys. Plasmas 12, 056310 (2005).

[4] J.-P. Davis, J. L. Brown, M. D. Knudson, and R. W. Lemke, J. Appl. Phys. 116, 204903 (2014).

[5] T. Ao, J. R. Asay, S. Chantrenne, M. R. Baer, and C. A. Hall, Rev. Sci. Instrum. 79, 013903 (2008).

[6] G. Avrillaud, L. Courtois, J. Guerre, P. L. Hereil, F. Lassalle, F. Bayol, P. L'Eplattenier, B. Kovalchuk, E. Kumpjak, N. Zoi, and A. Kim, in Proceedings of the 14th IEEE International Pulsed Power Conference edited by M. Giesselmann and A. Neuber (IEEE, Piscataway, 2003), p. 913.

[7] J. R. Woodworth, J. A. Alexander, F. R. Gruner, W. A. Stygar, M. J. Harden, J. R. Blickem, G. J. Dension, F. E. White, L. M. Lucero, H. D. Anderson, L. F. Bennett, S. F. Glover, D. Van DeValde, and M. G. Mazarakis, Phys. Rev. ST Accel. Beams 12, 060401 (2009).
[8] Y. M. Gupta, S. J. Turneaure, K. Perkins, K. Zimmerman, N. Arganbright, G. Shen, and P. Chow, Rev. Sci. Instrum. 83, 123905 (2012).

[9] M. L. Kiefer and M. M. Widner, in Proceedings of the 5th IEEE International Pulsed Power Conference, edited by M. F. Rose and P. J. Turchi (IEEE, Piscataway, NJ, 1985), p. 685.

[10] M. L. Kiefer, K. L. Shaw, K. W. Struve, M. M. Widner, and R. B. Spielman, SCREAMER, a pulsed power design tool, user's guide for version 3.2.4.2 (2008).

[11] COMSOL Multiphysics ${ }^{\circledR}$ 5.0.

[12] W. A. Stygar, T. C. Wagoner, H. C. Ives, Z. R. Wallace, V. Anaya, J. P. Corley, M. E. Cuneo, H. C. Harjes, J. A. Lott, G. R. Mowrer, E. A. Puetz, T. A. Thompson, S. E. Tripp, J. P. VanDevender, and J. R. Woodworth, Phys. Rev. ST Accel. Beams 9, 070401 (2006).

[13] W. A. Stygar, M. E. Cuneo, D. I. Headley, H. C. Ives, R. J. Leeper, M. G. Mazaratis, C. L. Olson, J. L. Porter, T. C. Wagoner, and J. R. Woodworth, Phys. Rev. ST Accel. Beams 10, 030401 (2007).

[14] E. M. Waisman and A. Wilson, J. Appl. Phys. 53, 731 (1982). 